\title{
Diagnostic Value of HMB-45 and Anti-Melan A Staining of Sentinel Lymph Nodes with Isolated Positive Cells
}

\author{
Muhammad N. Mahmood, M.D., Min W. Lee, M.D., Michael D. Linden, M.D., S.D. Nathanson M.D ., \\ Thomas J. Hornyak, M.D., Ph.D., Richard J. Zarbo, M.D., D.M.D. \\ Departments of Pathology (MNM, MWL, MDL, RJZ), General Surgery (SDN), and Dermatology (TJH), \\ Henry Ford Hospital, Detroit, Michigan
}

Numerous immunohistochemical stains have been employed to detect metastatic melanoma in sentinel lymph node (SLN) biopsies. HMB-45 is considered by some as a specific tool to detect early metastatic melanoma (1). Occasionally, one or two isolated HMB-45-positive cells may cause complications in diagnostic interpretation. The goal of this study was to evaluate the reliability of HMB-45 staining of SLNs with sparse isolated positive cells and to compare its staining with anti-Melan $A$ antibody. HMB-45 and anti-Melan $A$ antibody immunostaining was performed on (Group A) 15 histologically negative SLNs excised from patients with malignant melanoma (MM) and on (Group B) 15 histologically negative SLNs excised from patients with breast carcinoma (BC). None of the patients had clinical evidence of systemic metastasis at the time of SLN biopsy. Five cutaneous biopsies with changes of postinflammatory hyperpigmentation (PIHP) were also stained with both antibodies. HMB-45 staining was repeated in all Group B SLNs after blocking endogenous biotins. Electronmicroscopic studies were performed on all cases of PIHP. Isolated HMB-45-stained cells were present in 6 of 15 SLNs removed for MM; 8 of 15 for BC; and 3 of 5 cutaneous biopsies of PIHP. HMB-45 reactivity persisted after blocking endogenous biotins in 6 of 8 positive SLNs from Group B. Anti-Melan A antibody was negative in all SLNs of group $A$ and $B$ and in dermal melanophages of all five cases of PIHP. HMB-45 positivity was demonstrated in histologically negative SLNs and cutaneous biopsies, especially in the milieu of aggregated melanophages. Phagocytosis of premelanosomes by macrophages in the draining lymph nodes may account for iso-

\footnotetext{
Copyright () 2002 by The United States and Canadian Academy of Pathology, Inc.

VOL. 15, NO. 12, P. 1288, 2002 Printed in the U.S.A.

Date of acceptance: September 4, 2002.

Address reprint requests to: Muhammad N. Mahmood, M.D., Department of Pathology, Henry Ford Hospital, K-6, Clinic Building, 2799 West Grand Blvd., Detroit, MI 48202; e-mail: nosh97@hotmail.com; fax: 313-916-9433. DOI: 10.1097/01.MP.0000037313.33138.DF
}

lated cell positivity and can hinder correct diagnostic interpretation. HMB-45 may not be a reliable marker for the detection of micro-metastasis of MM and requires correlation with other immunohistochemical markers, such as anti-Melan A antibody, to enhance specificity.

KEY WORDS: Anti-Melan A, HMB-45, Melanophages, Metastatic melanoma, Sentinel lymph node.

Mod Pathol 2002;15(12):1288-1293

Metastasis to regional lymph nodes has significant therapeutic and prognostic implications for melanoma patients (2). The probability of lymph node metastasis is affected by factors like tumor thickness and depth of invasion by neoplastic cells $(3,4)$. In 1992, Morton and associates (5) introduced the surgical concept of sentinel lymph node biopsy for detection of early metastatic melanoma. The sentinel lymph node is presumed to be the nearest node to a primary melanoma and the most likely to become involved in metastatic disease. In selected patients, this technique of mapping tumor lymphatic drainage followed by lymph node biopsy is considered to be the surgical procedure of choice for staging metastatic melanoma. This method consists of injecting dye or radiolabeled tracer at the site of primary disease, mapping the lymphatic drainage, and detecting lymph nodes incorporating the injected tracer $(5,6)$. This procedure is considered to be beneficial for melanoma patients with clinically negative lymph nodes as it improves the chances of detecting early metastasis. A similar concept of sentinel node biopsy has also been applied in the diagnosis of metastatic breast carcinoma (7). One or more selected lymph nodes permits a more thorough and focused search for metastatic neoplastic cells. Hematoxylin and eosin ( $\mathrm{H} \& \mathrm{E})$-stained sections of these sentinel nodes, although essential, may miss occult or small foci of metastatic deposits (8). Tumor cells that are not apparent on $\mathrm{H} \&$ E-stained preparations may be detected using different immunohistochemical 
stains. Antibodies such as HMB-45, anti-Melan A, and against S-100 protein have been used individually or in combination to enhance the detection of "histologically negative" metastatic melanoma (8-10).

HMB-45 is a widely used immunohistochemical stain for detection of primary as well as metastatic melanoma (1). Presumably, this method uses monoclonal antibodies to a glycoprotein that is present in premelanosomes (11). In immunohistochemical assays, this antibody reacts with melanoma cells, junctional nevus cells, and fetal melanocytes but generally not with completely melanized normal adult melanocytes or with intradermal nevus cells (12-14). HMB-45 is nonreactive with almost all non-melanoma human malignancies, with the exception of rare tumors showing evidence of melanogenesis (e.g., pigmented schwannoma, clear cell sarcoma) or tumors associated with tuberous sclerosis complex (angiomyolipoma and lymphangiomyoma; 15, 27). Because of good resolution and low background staining, HMB-45 in sentinel lymph nodes is considered to have low false-positive results (1). Although this stain has good specificity, the sensitivity of HMB-45 for melanoma cells has been questioned by some authors $(16,17)$.

It has been noted that normal lymph nodes harness melanocytes in aggregates, particularly in juxtaposition to the lymph node capsule. Peripheral lymph nodes that drain skin are also known to harbor histiocytic cells containing melanin pigment (Fig. 1A). These histiocytic cells are referred to as melanophages. As seen in dermatopathic lymphadenopathy, melanophages are present in increased numbers in regional lymph nodes in association with certain skin disorders (18). The presence of one or two isolated HMB-45-positive cells may pose diagnostic difficulty in differentiating isolated melanoma cells from melanophages and benign cells showing melanocytic differentiation, such as nevus cells in lymph nodes (19). Our aims in the current investigation were to do the following: (1) determine the diagnostic value and reliability of HMB-45 staining in sentinel lymph nodes with one or two isolated positive cells and (2) compare HMB-45 with anti-Melan A antibody, another immunohistochemical marker employed for detection of melanoma cells.

\section{METHODS AND MATERIALS}

Thirty lymph nodes and five skin biopsies were included in this study. Lymph nodes comprised two groups. Group A included 15 patients with histologically negative sentinel lymph nodes removed for malignant melanoma; Group B included 15 patients with histologically negative sentinel lymph nodes removed for breast carcinoma. Lymph nodes from patients in Group B served as our control group. Five skin biopsies showing changes of postinflammatory hyperpigmentation were selected at random. All surgical procedures were performed from 1996 to 1999 at Henry Ford Hospital, Detroit, Michigan. All selected patients were clinically negative for metastatic disease.

Lymph nodes from both Groups A and B were fixed in $10 \%$ buffered formalin and entirely embedded in paraffin blocks. Multiple slides were obtained for $\mathrm{H} \& \mathrm{E}$ sections and immunohistochemical analysis. Three slides per case were stained with $\mathrm{H} \&$ E. All cases were stained with HMB-45 (Gown; Phenopath Lab., Seattle, WA; 1:10000 dilution) and anti-Melan A antibody (DAKO, Carpinteria, CA; clone A103, 1:50 dilution). All HMB-45-positive cases in Group B (8/15) were stained again with HMB-45 after blocking endogenous biotins using an avidin-biotin blocking system (DAKO; Carpinteria, CA). Five skin biopsies of postinflammatory hyperpigmentation were also stained using HMB-45 and anti-Melan A antibody. Formalin fixed and paraffin embedded tissues from all five skin biopsy cases were reprocessed for electron-microscopic study. All immunohistochemical stains were performed using a labeled streptavidin-biotin (DAKO) method with DAKO automated immunostainer. 3-Amino-9 ethylcarbazole (AEC) was used for chromogen working solution to distinguish positive stain from preexisting melanin. Positive and negative controls were used for each antibody.

\section{RESULTS}

H \& E sections of all 30 lymph nodes were negative for metastatic disease. None of these lymph nodes showed inclusions of melanocytic nevus cells. Skin biopsies showed prominent melanin pigmentation in dermal melanophages with variable amount of melanin in the basal layer (Fig. 1B). Review of HMB-45-stained sections of Group A showed isolated scattered positive cells in 6 of 15 lymph nodes (40\%; Fig. 1C). HMB-45-stained sections of Group B showed isolated scattered positive cells in 8 of 15 lymph nodes (53.3\%; Fig. 1D). HMB-45 positivity persisted after blocking endogenous biotins in 6 of 8 lymph nodes in Group B. The HMB-45-positive cells, in both groups, were randomly distributed in the cortex and often closely associated with small aggregates of melanin containing histiocytes (melanophages). It was difficult to evaluate the nuclear characteristics of HMB-45positive cells with certainty because of strong cytoplasmic stain blurring the nuclear outline. Nevertheless, many of the HMB-45-positive cells were recognized as histiocytic cells. Anti-Melan A immu- 
nostaining was negative in all lymph nodes from Group A and B. None of the isolated HMB-45positive cells showed immunoreactivity with antiMelan A antibody.

Three of five skin biopsies showed isolated HMB45-positive cells in the papillary dermis (Fig. 1E). These positive cells were readily recognized as melanophages. Anti-Melan A showed negativity in HMB-45-positive dermal melanophages in all five cases, although few mature melanocytes in the basal layer of the epidermis did show positive staining. As we used AEC for chromogen, positive stain was easily distinguished from cytoplasmic melanin because of different tinctorial quality. Electronmicroscopic analysis of these HMB-45-positive histiocytic cells in the dermis revealed melanosomes within phagolysosomes (Fig. 1F). Most of the melanosomes were fully melanized, recognized as Stage IV melanosomes, but occasional Stage II-III melanosomes were also recognized.

All 30 patients had no clinical evidence of metastatic disease at the time of sentinel lymph node biopsy. Of patients in Group A, four had primary lesions on the forearm, three on the back, three on the forehead, two on the calf, and two on the shoulder; and one patient had primary location of melanoma on the earlobe. Seven patients had primary melanoma of Clark's Level III with Breslow thickness ranging from 0.3 to $1.9 \mathrm{~mm}$ (mean, $1.2 \mathrm{~mm}$ ), and eight patients had primaries of Clark's Level IV with Breslow thickness ranging from 0.7 to $4.9 \mathrm{~mm}$ (mean, $2.3 \mathrm{~mm}$ ). Patients in Group A had a mean age of 42.3 years; 13 were Caucasians and 2 were African Americans. Similarly, patients in Group B had a mean age of 48.2 years; 9 were Caucasians and 6 were African Americans. Clinical follow-up was performed starting from the time of primary diagnosis to commencement of this study. All patients chosen in Group A were disease free without any evidence of metastasis or recurrence of melanoma (mean follow-up, 2.3 y). No patient in Group $\mathrm{B}$ had any known clinical history of primary cutaneous melanoma.

\section{DISCUSSION}

Because of the prognostic implications of lymph node metastasis of malignant melanoma, there is a continuous effort to refine the technique and criteria involved in the detection of this stage of disease. Identification of metastatic melanoma in sentinel lymph nodes can be carried out without difficulty on $\mathrm{H}$ \& E-stained sections when there are cohesive cell nests or clusters of neoplastic cells present. However, early metastatic disease can occur in the form of single neoplastic cells, which can create difficulties in light-microscopic detection on $\mathrm{H} \& \mathrm{E}$ sections. There is an ongoing quest for better and more precise ways of detecting and confirming isolated melanoma cells in early metastasis. Numerous antibodies directed against different components in malignant melanoma cells have been employed to facilitate the diagnosis $(1,17,25,28-$ 30). Various recent investigations have suggested supremacy of one type of immunohistochemical stain over another. Messina et al. (17) claimed S-100 to be extremely sensitive and suggested HMB-45 to be too insensitive. However, S-100 positivity was also demonstrated in dendritic reticulum cells and Langerhans cells in addition to melanoma cells in the lymph nodes, suggesting less than optimal specificity of this technique. Baisden et al. (1) considered HMB-45 to be a more reliable and specific marker of melanoma cells because of higher clarity and minimal background staining in lymph node biopsies. Gibbs et al. (10) concluded that combination of multiple levels of $\mathrm{H} \& \mathrm{E}$ sections in conjunction with S-100 and HMB-45 immunohistochemical staining dramatically increases the overall diagnostic sensitivity of sentinel lymph node biopsy. The usage of S-100 staining, which is known to stain almost $100 \%$ of melanomas, partially compensates for the lower sensitivity of HMB-45. However, as $\mathrm{S}-100$ expression is not melanocyte specific, an S-100-positive and HMB-45-negative cluster of cells can be doubtful. Because other cell populations in lymph nodes are positive for S-100 protein, it appears to be an unreliable stain when single-cell metastasis is under consideration. As there is no clear consensus on this issue, many laboratories usually employ a panel of stains. To make interpretation even more difficult, a dilemma arises when widely spaced isolated positive cells are found on stained sections. In a study by Hatta et al. (19), HMB-45-positive cells seen in histologically negative lymph nodes were found to occur singly and in a dispersed fashion. Determining the character of these single positive isolated cells can be problematic and may eventuate in up-staging of nodenegative patients. These widely variable opinions and lack of clear consensus on this important issue prompted us to study HMB-45 staining in sentinel lymph node biopsies.

Our results showed comparable HMB-45 isolated cell positivity between lymph nodes removed for metastatic melanoma and breast carcinoma. Staining was particularly obvious in the vicinity of aggregated melanophages. Skin tissue from postinflammatory hyperpigmentation contained histologically and electron-microscopically identifiable melanophages. These melanophages also showed positivity with HMB-45. In an effort to reduce background staining in sentinel lymph nodes, we blocked endogenous biotin activity, but staining persisted in nearly all cells. We believe that the phagocytosis of premelanosomes 

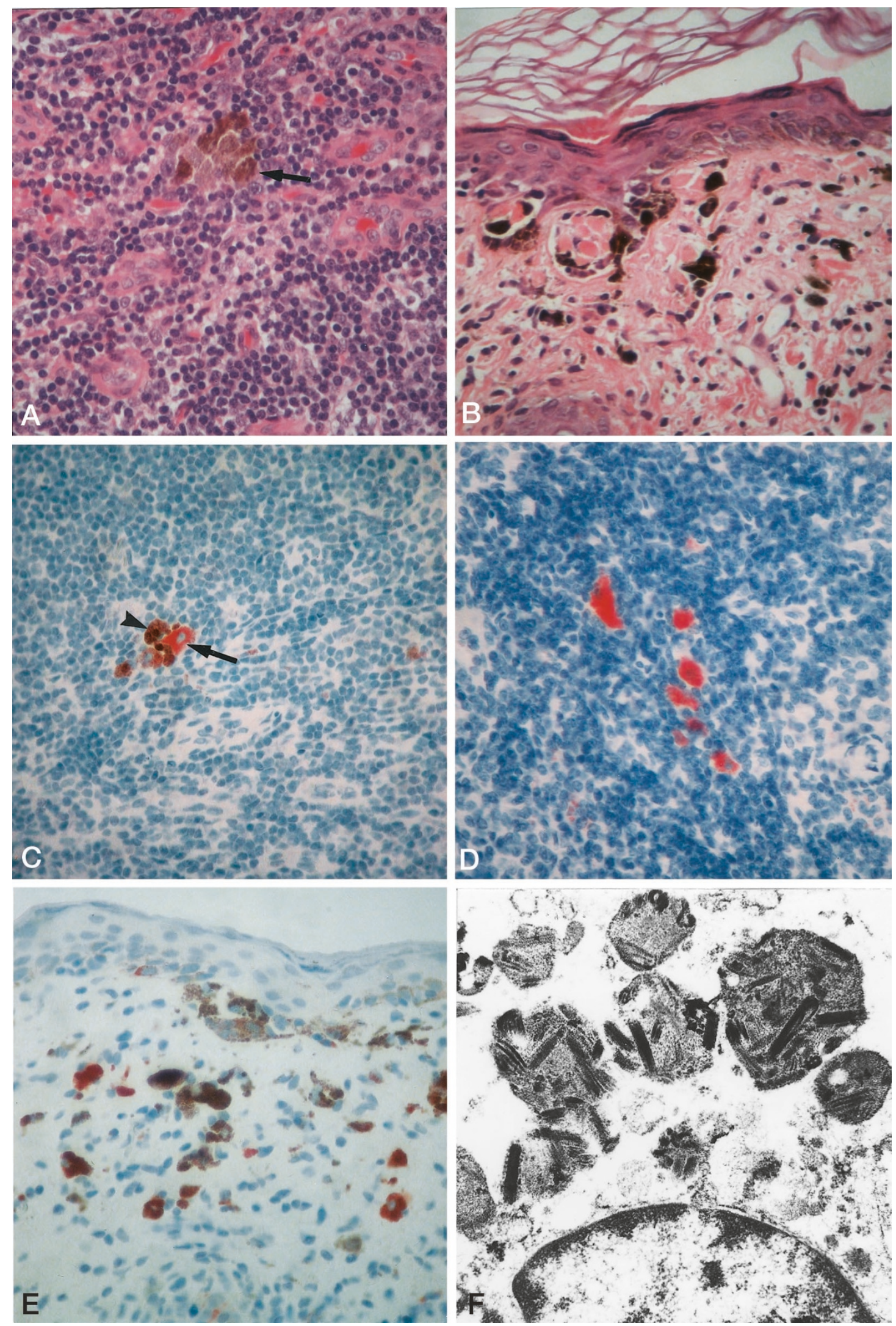

FIGURE 1. A, Melanophages seen in aggregates (arrow) in a sentinel lymph node draining skin and excised for staging of metastatic melanoma (original magnification, $200 \times$ ). B, Skin biopsy with dermal melanophages showing prominent melanin incontinence (original magnification, 100 $\times$ ). C, HMB-45-positive cells in lymph nodes removed for malignant melanoma (arrow). Adjacent melanin containing histiocytic cells (arrowhead) are also readily recognized on $\mathrm{H} \& \mathrm{E}$ sections (original magnification, 100×). D, HMB-45-positive cells in a sentinel lymph node excised for breast carcinoma (original magnification, $100 \times$ ). E, HMB-45-positive cells seen in melanophages present in papillary dermis of skin biopsies from patients with postinflammatory hyperpigmentation (original magnification, 100×). F, Premelanosomes in dermal melanophages in skin biopsy of postinflammatory hyperpigmentation (original magnification, $22,500 \times$ ). 
by histiocytic cells in the draining lymph nodes and dermis may be responsible for the reactivity of these cells. HMB-45 stains melanophages and does not appear to provide a confident interpretation of metastasis when isolated positive cells are seen. This observation is most meaningful in cases in which a question of isolated melanoma cells versus benign melanophages is under consideration.

Furthermore, we performed anti-Melan A antibody (clone A103) immunostain on all sentinel lymph nodes. Anti-Melan A is another more recently described antibody useful in the diagnosis of primary and metastatic melanoma. The Melan A gene, cloned by Coulie et al. (20) from a human melanoma cell line, encodes a melanoma-specific antigen recognized by autologous cytotoxic lymphocytes. In structure, Melan A antigen is a transmembrane protein composed of 118 amino acids and is expressed in a majority of human melanomas (21-23). With the exception of retina, Melan A RNA has not been detected in other normal human tissues $(24,25)$. Recognition of Melan A paved the way for investigations of similar melanoma antigens recognized by autologous T-lymphocytes. Among these are MAGE 1, MAGE 3, tyrosinase, gp100, gp75, BAGE 1, and GAGE 1 (31, 32). For immunohistochemical purposes, A103 clone is the recombinant immunogen corresponding to Melan A. A103 antibody recognizes a $20-$ to $22-\mathrm{kDa}$ doublet in a Melan A mRNA-positive melanoma cell line (20). Chen et al. (26) detected no reactivity of this antibody in Melan A mRNA-negative cell lines. Anti-Melan A, clone A103, shows uniform cytoplasmic staining in a high percentage of primary human melanomas (>90\%; 26). Jungbluth et al. (25), from their comparative study, concluded that A103 could be used as a first-line antibody in the diagnosis of metastatic melanoma. A103 showed homogeneous staining in a significantly higher proportion of cases of malignant melanoma than did HMB-45 (72\% versus $52 \%$; 25). In our study, we saw no significant reactivity when histologically negative lymph nodes were stained with A103. This contrasts with the pattern of staining seen with HMB45 , in which multiple lymph nodes showed isolated positive cells. The fact that Melan A is negative in all HMB-45-positive melanophages in lymph nodes and skin biopsies makes it a valuable diagnostic adjunct, particularly in cases in which single isolated melanophages mimic micrometastasis.

Acknowledgment: The authors specially thank the department of immunohistochemistry, Henry Ford Hospital, for providing excellent technical support for this work.

\section{REFERENCES}

1. Baisden BL, Askin FB, Lange JR, Westra WH. HMB-45 immunohistochemical staining of sentinel lymph nodes. A specific method for enhancing detection of micrometastases in patients with melanoma. Am J Surg Pathol 2000;24:1140-6.

2. Robert ME, Wen DR, Cochran AJ. Pathological evaluation of the regional lymph nodes in malignant melanoma. Semin Diagn Pathol 1993;10:102-15.

3. Balch CM, Murad TM, Soong SJ. Tumor thickness as a guide to surgical management of clinical stage I melanoma patients. Cancer 1975;35:666-76.

4. Lee YTN. Diagnosis, treatment and prognosis of early melanoma. The importance of depth of microinvasion. Ann Surg 1980;191:87-97.

5. Morton DL, Wen DR, Wong JH. Technical details of intraoperative lymphatic mapping for early stage melanoma. Arch Surg 1992;127:392-9.

6. Reintgen D, Cruse CW, Wells K. The orderly progression of melanoma nodal metastasis. Ann Surg 1994;220:759-67.

7. McMaster KM, Tuttle TM, Carlson DJ, Brown CM. Sentinel lymph node biopsy for breast cancer a suitable alternative to routine axillary dissection in multi-institutional practice when optimal technique is used. J Clin Oncol 2000;18:2560-6.

8. Cochran AJ, Wen DR, Morton DL. Occult tumor cells in the lymph nodes of patients with pathological stage I malignant melanoma. An immunohistochemical study. Am J Surg Pathol 1988;12:612-8.

9. Cochran AJ, Wen DR, Herschman HR. Occult melanoma in lymph nodes detected by antiserum to S-100 protein. Int J Cancer 1984;34:159-63.

10. Gibbs JF, Huang PP, Zhang PJ, Kraybill WG, Cheney R. Accuracy of pathologic techniques for the diagnosis of metastatic melanoma in sentinel lymph nodes. Ann Surg Oncol 1999;6:699-704.

11. Schaumburg-Lever G. New applications of electron microscopy techniques in dermatopathology. J Cutan Pathol 1995; 22:483-7.

12. Gown AM, Vogel AM, Hoak D, Gough F, Mcnutt MA. Monoclonal antibodies specific for melanocytic tumors distinguish subpopulations of melanocytes. Am J Pathol 1986;123: 195-203.

13. Colombari R, Bonetti F, Zamboni G. Distribution of melanoma specific antibody(HMB-45) in benign and malignant melanocytic tumors. Virchows Arch A Pathol Anat 1988;413: 17-24.

14. Holbrook KA, Underwood RA, Vogel AM. The appearance, density and distribution of melanocytes in human embryonic and fetal skin revealed by anti-melanoma antibody HMB-45. Anal Embryol 1989;180:443-5.

15. Bonetti F, Pea M, Martignoni G. False positive immunostaining of normal epithelia and carcinomas with ascites fluid preparations of anti-melanoma monoclonal antibody HMB45. Am J Clin Pathol 1991;95:454-9.

16. Achilles E, Schroder S. Positive cytokeratin results in malignant melanoma. Pitfall in differential immunohistological diagnosis of occult neoplasms. Pathologe 1994;15:235-41.

17. Messina JL, Glass F, Cruse CW, Berman C, Ku NK, Reintgen DS. Pathologic examination of the sentinel lymph node in malignant melanoma. Am J Surg Pathol 1999;23:686-90.

18. Scheffer E, Meijer CJLM, Van Volten WA. Dermatopathic lymphadenopathy and lymph node involvement in mycosis fungoides. Cancer 1980;45:137-48.

19. Hatta N, Takata M, Takehara K, Ohara K. Polymerase chain reaction and immunohistochemistry frequently detect occult melanoma cells in regional lymph nodes of melanoma patients. J Clin Pathol 1998;51:597-601.

20. Coulie PG, Brichard V, Van Pal A, Wolfel T, Schneider J, Traversari C. A new gene coding for a differentiation antigen 
recognized by autologous cytolytic $\mathrm{T}$ lymphocytes on HLA-A2 melanomas. J Exp Med 1994;180:35-42.

21. Fetsch PA, Cormier J, Hijazi YM. Immunocytochemical detection of MART-1 in fresh and paraffin embedded malignant melanomas. J Immunother 1997;20:60-4.

22. Kawakami Y, Battles JK, Kobayashi T, Ennis W, Wang X, Tupesis JP, et al. Production of recombinant MART-1 proteins and specific anti-MART-1 polyclonal and monoclonal antibodies: use in the characterization of the human melanoma antigen MART-1. J Immunol Methods 1997;202:13-25.

23. Sarantou T, Chi DDJ, Garrison DA, Conrad AJ, Schmid P, Morton DL. Melanoma-associated antigens as messenger RNA detection markers for melanoma. Cancer Res 1997;57: 1371-6.

24. Kawakami Y, Eliyahu S, Delgado CH, Robbins PF, Sakaguchi $\mathrm{K}$, Apella E. Identification of a human melanoma antigen recognized by tumor infiltrating lymphocytes associated with in vivo tumor rejection. Proc Natl Acad Sci U S A 1994;91:6458-62.

25. Jungbluth A, Bausam K, Gerald W, Stockert E, Coplan K, Iversen K. A103: an anti-Melan-A monoclonal antibody for the detection of malignant melanoma in paraffin-embedded tissues. Am J Surg Pathol 1998;22:595-602.

26. Chen YT, Stockert E, Jungbluth A, Tsang S, Coplan KA, Scanlan MJ. Serological analysis of Melan-A, a melanocyte- specific protein homogeneously expressed in human melanomas. Proc Natl Acad Sci U S A 1996;93:5915-9.

27. Gaffey MJ, Mills SE, Askin FB, Ross GW, Sale GE, Kulander BG, et al. Clear cell tumor of the lung. A clinicopathologic, immunohistochemical, and ultrastructural study of eight cases. Am J Surg Pathol 1990;14:248-59.

28. Van Duinen SG, Ruiter DJ, Scheffer E. A staining procedure for melanin in semi thin and ultra thin epoxy sections. Histopathology 1986;7:35-48.

29. Thompson W, Mackie RM. Comparison of five antimelanoma antibodies for identification of melanocytic cells on tissue sections in routine dermatopathology. J Am Acad Dermatol 1989;21:1280-4.

30. Fernando SSE, Johnson S, Bate J. Immunohistochemical analysis of cutaneous malignant melanoma: comparison of S-100 protein, HMB-45 monoclonal antibody and NK/C3 monoclonal antibody. Pathology 1994;26:16-9.

31. Kawakami Y, Bang N, Wang X, Rosenberg SA. Recognition of shared melanoma antigens in association with major HLA-A alleles by tumor infiltrating T-lymphocytes from 123 patients with melanoma. J Immunother 2000;23:17-27.

32. Vijayasaradhi S, Xu Y, Bouchard B, Houghton AN. Intracellular sorting and targeting of melanosomal membrane proteins: identification of signals for sorting of the human locus protein gp 75. J Cell Biol 1995;130:807-20. 